\title{
Treatment timing and multidisciplinary approach in Apert syndrome
}

\author{
MariaTeresa Fadda, MD, DDS, PhD ${ }^{1}$ \\ Gaetano lerardo, DDS, PhD² \\ Barbara Ladniak, DDS, $\mathrm{PhD}^{2}$ \\ Gianni Di Giorgio, DDS, $\mathrm{PhD}^{2}$ \\ Alessandro Caporlingua MD ${ }^{1}$ \\ Ingrid Raponi, MD ${ }^{1}$ \\ Alessandro Silvestri, MD, DDS ${ }^{3}$
}

Group of Apert syndrome, Policlinico Umberto I, "Sapienza" University of Rome, Italy

1 Department of Oral and Maxillofacial Sciences, Maxillofacial Surgery Unit, Policlinico Umberto I, "Sapienza" University of Rome, Italy

2 Department of Oral and Maxillofacial Sciences, Pediatric Unit, "Sapienza" University of Rome, Italy

3 Department of Oral and Maxillofacial Sciences, Orthognathodontic Unit, "Sapienza" University of Rome, Italy

Collaborators: A. Polimeni ${ }^{4}$, M. Bossù 4 , E. Barbato 4 , G. lerardo ${ }^{4}$, A. Silvestri' ${ }^{4}$ A. Pizzuti ${ }^{4}$, A. De Luca 4 , V. Guida $^{4}$, A. Giancotti ${ }^{4}$, C. Moretti ${ }^{4}$, P. Papoff ${ }^{4}$, A. Spalice ${ }^{4}$, F. Ursitti ${ }^{4}$, G. Gualdi ${ }^{4}$, C. Di Biasi ${ }^{4}$, C. Andreoli ${ }^{4}$, C. Colaiacono ${ }^{4}$, R. Delfini ${ }^{4}$, A. Santoro ${ }^{4}$, A. Caporlingua $^{4}$, F. Caporlingua 4 , M. De Vincentis ${ }^{4}$, A. Greco ${ }^{4}$ P. Cascone ${ }^{4}$, V. Valentini ${ }^{4}$, M.T. Fadda ${ }^{4}$, C. Ungari ${ }^{4}$, M. Gharbiya ${ }^{4}$, M. Marenco ${ }^{4}$, B. Carlesimo ${ }^{4}$, A. Spagnoli $^{4}$, V. Mazzone ${ }^{4}$, M. Amabili ${ }^{4}$, L. Silvestrini ${ }^{4}$

Corresponding author:

Maria Teresa Fadda

Department of Oral and Maxillofacial Sciences,

Maxillofacial Surgery Unit, Policlinico Umberto I

"Sapienza" University of Rome

Via Caserta 6

00161 Rome, Italy

E-mail: mariateresa.fadda@uniroma1.it

\section{Summary}

Apert syndrome is a rare congenital disorder characterized by craniosynostosis, midface hypoplasia and symmetric syndactyly of hands and feet. Abnormalities associated with Apert syndrome include premature fusion of coronal sutures system (coronal sutures and less frequently lambdoid suture) resulting in brachiturricephalic dismorphism and impaired skull base growth.

After this brief explanation it is clear that these anatomical abnormalities may have a negative impact on the ability to perform essential functions. Due to the complexity of the syndrome a multidisciplinary (respiratory, cerebral, maxillo-mandibular, dental, ophthalmic and orthopaedic) approach is necessary in treating the psychological, aesthetic and functional issues. The aim of this paper is to analyse the different functional issues and surgical methods trying to enhance results through a treatment plan which includes different specialities involved in Apert syndrome treatment. Reduced intellectual capacity is associated to the high number of general anaesthesia the small patients are subject to. Therefore the diagnostic and therapeutic treatment plan in these patients has established integrated and tailored surgical procedures based on the patients' age in order to reduce the number of general anaesthesia, thus simplifying therapy for both Apert patients and their family members.

Key words: Apert syndrome, multidisciplinary approach, congenital disorders.

\section{Introduction}

Apert syndrome is a rare congenital disorder characterized by craniosynostosis, midface hypoplasia and symmetric syndactyly of hands and feet. Apert syndrome was first reported by Wheaton in 1894 and a French paediatrician, Eugene Apert, published a series of nine cases in 1906.

Apert syndrome is estimated to affect 1 in 160,000 live births. It is most frequently caused by a de novo mutation in the male gameter. Two missense mutations in the fibroblast growth factor receptor 2 (FGFR2) gene on chromosome 10 (1), have been found to account for the disorder in approximately $98 \%$ of patients (1): the p.P253R mutation accounts for 33\% of the cases and is associated with more severe syndactyly when compared to other mutations that cause Apert syndrome: p.S252W, which accounts for $66 \%$ of cases is strongly associated with cleft palate (2). These mutations lead to loss of ligand specificity of receptor, causing abnormalities in extracellular matrix composition and premature calvarial ossification. The syndrome may be inherited in an autosomal dominant trait. In these cases, advanced parental age is often associated with a higher risk in having an affected child (3). With the present work we emphasize the need of an integrated and multispecialistic approach to the syndrome. We want to define main therapeutic steps performed by our group on patients affected by Apert syndrome. 


\section{Clinical features}

Abnormalities associated with Apert syndrome include premature fusion of the coronal sutures system (coronal sutures and less frequently lambdoid suture) resulting in brachiturricephalic dismorphism and impaired growth of the skull base. When the lambdoid suture is involved, the resulting underdevelopment of the posterior cranial fossa may evolve toward an Arnold-Chiari malformation with chronic tonsillar herniation and increased risk of hydrocephalus (4). Hydrocephalus is associated with raised intracranial pressure leading to clinical manifestations such as somnolence, irritability, vomiting, sixth cranial nerve palsy and cephalalgia. Other typical findings are malformations of brain gyri and heterotopia of the gray matter, ventriculomegaly, the malformations of limbic structures and an ipoplastic or even absent corpus callosum (5), absence or cystric septum. Patients may present or develop intervertebral fusion, mainly involving the cervical spine.

In the orbital region there are retrusion of the upper or lower orbital margin, associated with hypertelorism, eyelid antimongoloid, proptosis, strabismus and eyebrow cleft (Fig. 1). Orbital retrusion in Apert syndrome may extend to the midface, causing maxillary hypoplasia with a V-Shaped maxillary dental arch (6). An anterior open bite with Angle class III malocclusion is also common. The palate is high and narrow with lateral hypertrophic swellings (Fig. 2). Clefting of the soft palate or uvula occurs in $30 \%$ of patients. Delayed or ectopic tooth eruption, cross bite and dental crowding are common (7). The nose has a flattened dorsum and a small bulbous tip.

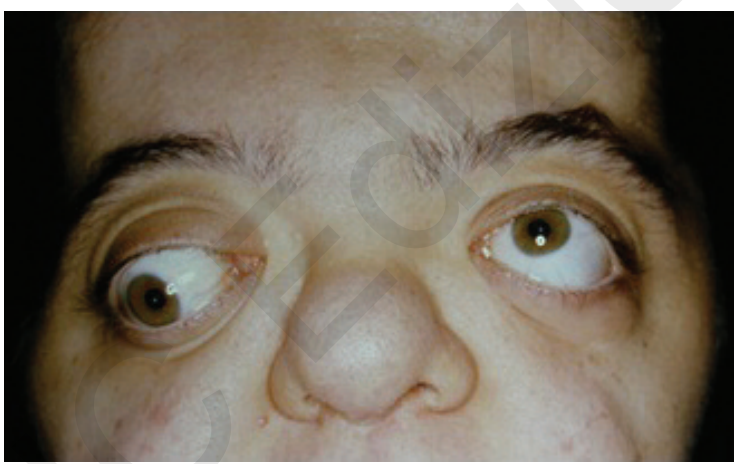

Figure 1. Orbital dystopia, hypertelorism, eyelid antimongoloid and globous nose in a patient affected by Apert syndrome.

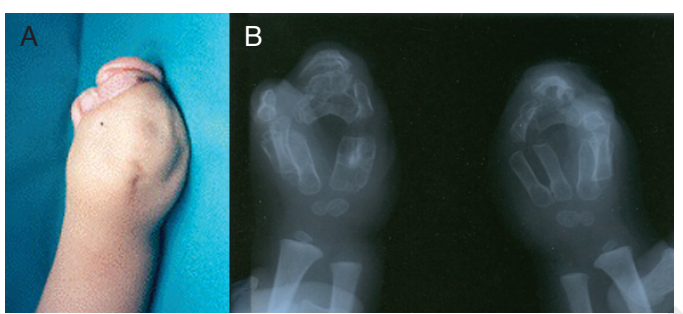

Figure 3. A and B Severe syndactyly of a hand with complete fusion of all five digits at the level of the terminal and middle phalanges, the hand has a cup-like appearance.

In addition to midface hypoplasia, the pharynx is small and the bony nasal cavity is narrowed. These abnormalities may cause severe respiratory distress, especially during the neonatal period when patients are nasal breathers. Therefore most neonates require airway interventions such as tracheostomy. Older patients should be screened with polisomnograms for obstructive sleep apnea (8). Conductive hearing loss is common in Apert syndrome. This results from chronic otitis media and occasionally from middle ear anomalies (9). The ears are set low and may be enlarged. Bilateral symmetrical complex syndactyly of hands and feet always occur with this syndrome. The hands have the following four common features regardless of severity (Fig. 3):

1. Complex syndactyly exists between the index, middle and ring fingers

2. The thumb is shortened, with the proximal or distal segment deviated radially (clinodactyly)

3. The fourth interdigital space exhibits simple syndactyly

4. The phalanges exhibit brachyphalagism.

At birth the cuneiform bones are well segmented. Progressive fusions interest the tarsal fist, but during childhood it will progressively involve the metatarsal shafts and eventually involve fusions between distal and proximal phalanges of toes.

Most patients with Apert syndrome have neurologic involvement. Slow intellectual development occur in approximately $50 \%$ of cases, however most of these patients have only mild intellectual deficiency.

\section{Treatment plan}

After this brief explanation it is clear that these anatomical abnormalities may have a negative impact on the ability to perform essential functions.

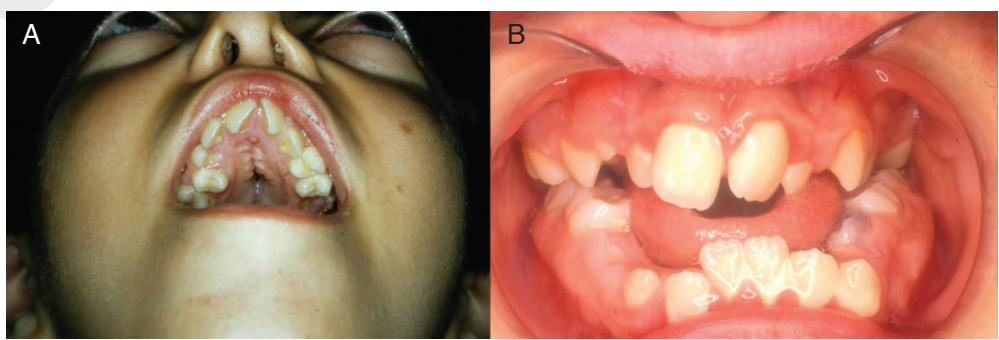

Figure 2. Intraoral features in Apert syndrome. A: cleft palate, V-Shaped maxillary dental arch, shovel-shaped incisors; B: open bite, Angle class III malocclusion, crossbite, dental crowding, eruption anomalies. 
Due to the complexity of the syndrome a multidisciplinary (respiratory, cerebral, maxillo-mandibular, dental, ophthalmic and orthopaedic) approach is necessary in treating the psychological, aesthetic and functional issues.

We believe that the best functional and aesthetic results can be achieved only through an integrated multidisciplinary approach on the bases of the experience gained at the centers of cranio-facial surgery. Other considerations deal with high number of operations that must be performed at an early age, during growth and at the end of development. It has been shown that the high number of general anaesthesia in small patients is to be correlated with intellectual limitations and with the offset of cognitive impairment. This data gives the chance to reflect on both, the need to anticipate or postpone the planned measures and on the need to perform multiple operations at the same time to reduce the number of general anaesthesia. The aim of the paper is to analyse the different functional problems and the different surgical methods trying to optimize results according to a timetable that provides the integration of different specialities involved in the Apert syndrome treatment.

We decided to divide treatment plan in three steps:

- $\quad$ step 1 - from birth to age 2

- $\quad$ step 2 - growth period

- $\quad$ step 3 - adult age.

The latter will be described for each age group with relative diagnostic and treatment options in order to optimize the protocol.

\section{From birth to age 2}

At birth and in the first weeks cerebral, respiratory and ocular bulb emergencies should be taken into account, particularly in severe cases of Apert syndrome. For these patients immediate surgical treatment is required in order to prevent or correct papilledema, corneal ulcers, severe respiratory distress and intercranial hypertension. It is fundamental to start integrated counselling with all medical team involved in the diagnosis and treatment protocol: genetists, neuropediatricians, pediatricians, maxillo-facial surgeons, neurosurgeons, hand surgeons, ophthalmologists. Working together they guarantee a valid and multidisciplinary support to Apert families.

Surgery in Apert cases is important with respect to patient's age, the degree of case-specific craniofacial dysmorphism and neuro-anatomical deformity. In this period cranioplasty and/or posterior vault expansion associated with a fronto-orbital advancement (Fig. 4) should be performed in order to guarantee the physiological vault expansion.

Surgical options and timing are still debated in literature: Fearon and Podner prefer to post-pone cranial vault expansion to the $15^{\text {th }}$ month in cases of non adverse clinical features and at 5-9 years for second cranial vault expansion with separate Le Fort III distraction, if necessary (5).

Allam et al., at the Los Angeles medical center, prefer to perform frontal-orbital advancement at 4-6 months of age, while posterior vault expansion is performed at 6-12 months. Monobloc advancement or Le Fort III osteotomy and facial bipartition (Fig. 5) are performed at 6-7 years (10).

Oberoi et al. advice to perform frontal-orbital advancement at 6-12 months and midface Le Fort III advancement at 9-12 years (11). Correction of syndactyly treatment is recommended at 13 months (12) for separation of the finger to gain manipulation movement. Cleft palate repair should be performed in this period to rehabilitate speech and swallow disorders.

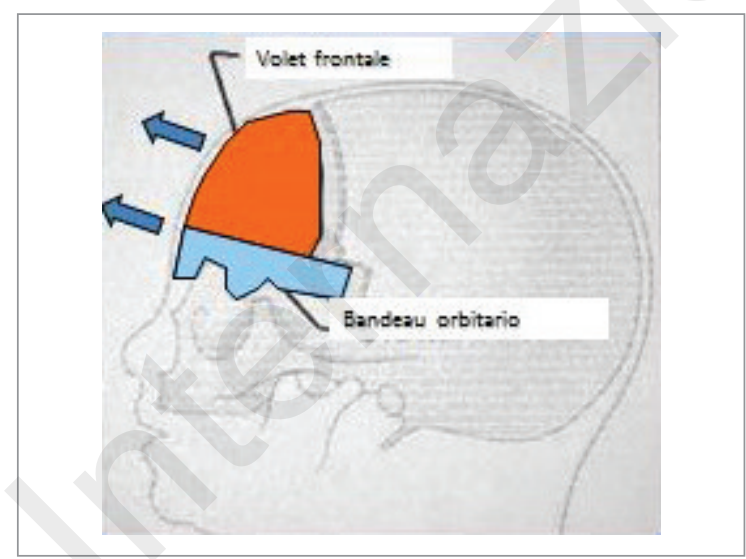

Figure 4. Fronto-orbital advancement.

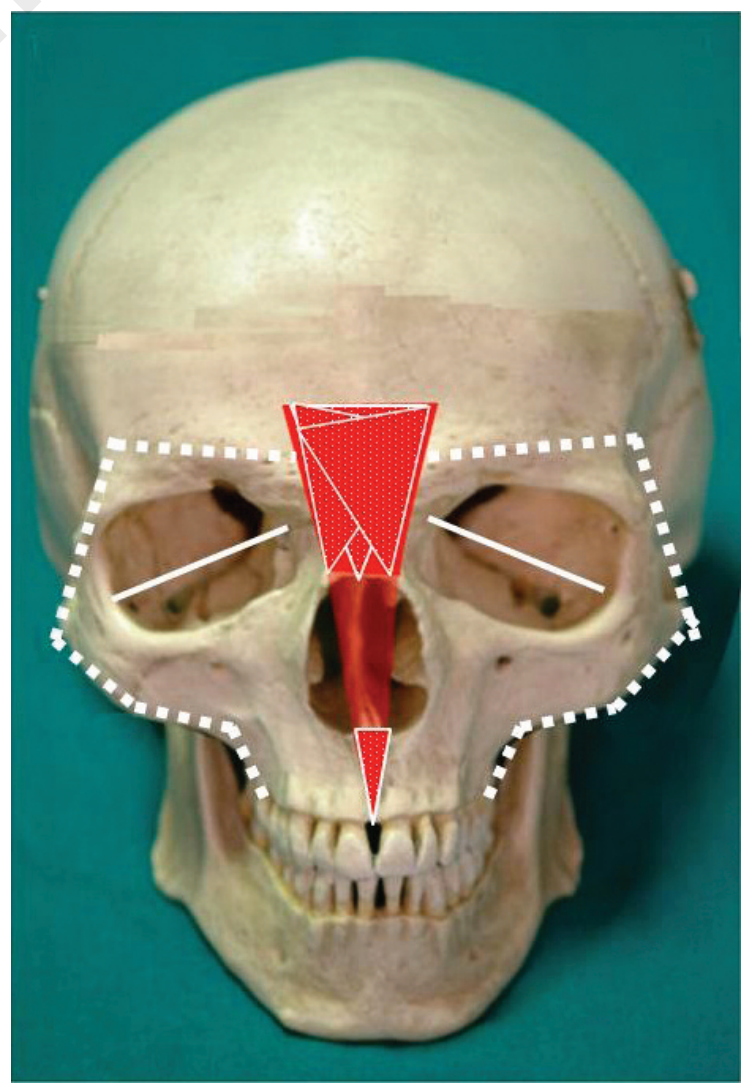

Figure 5. Facial bipartition. 


\section{Growth Period}

During growth (up to age 12) both the transversal dimension of the orbital region (hypertelorism and antimongolodin slant of the palpebral fissure) and retrusion of the middle third must be corrected. In the first case surgeons may perform an orbital mobilization. In the second case a Le Fort III osteotomy with external distraction can be performed (Fig. 6). Facial bipartition by external distraction may be performed (13-15) with good results, hypertelorism and midface flattening are associated (Fig. 7).

Since growth of the midface (especially in Apert syndrome) is not predictable, sometimes distraction advancement should be repeated at a later age to catch up with mandibular growth. Thus we believe that midface advancement should be delayed at a later age in order to obtain predictable results and to reduce the surgical load.

If the patient has adequate projection of the superior orbital ridge, Le Fort III osteotomy is the chosen procedure, whereas monobloc advancement, which advances the forehead simultaneously with the midface, may produce better results if the forehead remains retruded (16). Le Fort III or monobloc distraction provide greater ad- vancement than traditional advancement and may reduce complications such as meningitis.

For our patients treatment we always use an external distraction device (Fig. 6B-7). In the past internal distractions were widely used (Fig. 6C), however from our experience and in accordance with literature (8, 17 ), the use of an external halo device offers greater advancement and better vector control.

Between 2 and 7 years, it is essential to follow cognitive and language development especially after cleft repair. This is particularly beneficial for rehabilitation with lingual devices.

In addition during this period the following must be performed:

- eye surgery, correction of strabismus after the final positioning of the orbits

- hand and foot surgery preferably after 4-5 years

- dental and orthodontic therapies to monitor teeth eruptions, prevent caries, guide the eruption and allow dental alignment.

Hand, eye and dental surgery can be carried out simultaneously in order to reduce the number of general anaesthesia to which the patient must be exposed. Oral clinical features in Apert syndrome are: maxillary ipoplasia, bifid uvula, Byzantine-arch palate associat-
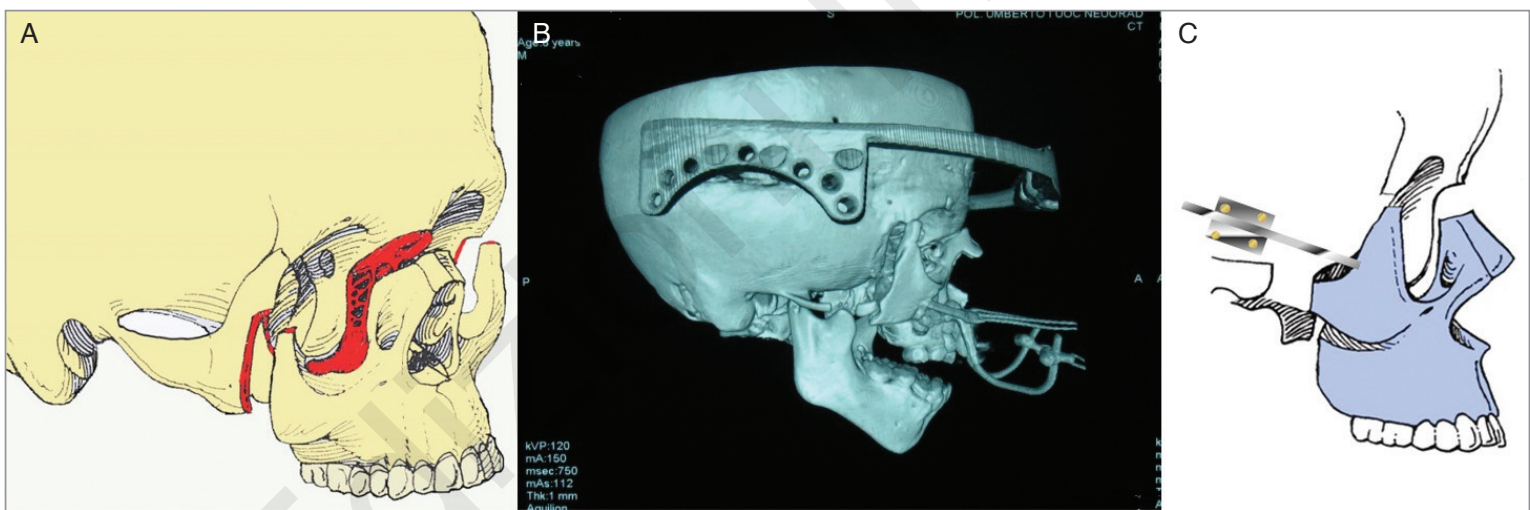

Figure 6. Middle third advancement A:Le Fort III osteotomy line; B: external distractor device applied in a patients after a Le Fort III procedure. C: internal distractor device applied in a patient after Le Fort III osteotomies.

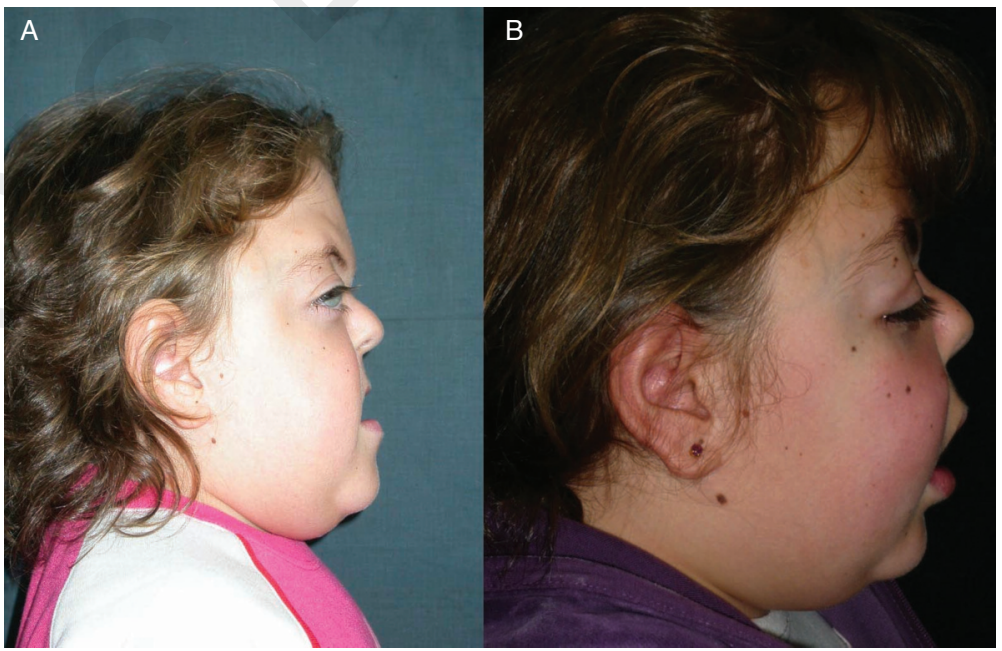

Figure 7. Third middle advancement in a 8 year-old child with external distraction. A: Pre surgical, B: Post surgical outcome. 
ed with lateral swellings of the palatine process, dental plaque, dental calculus, congestion and swelling of the gingival and periodontal pseudo pockets, maxillary dental crowding, shovel-shaped incisors, dental agenesis and early dental loss (18).

In literature despite big interest in genetics and treatment of the syndrome, today no emphasis is given to prevention and endodontic-conservative treatment. Often because of the patients' hand malformations unsatisfactory oral care and dental problems occur. Early dental treatment consists in instructions for both patient and parents. It is important in our opinion to communicate the importance of avoiding dental plaque, carious process that may cause peridontitis, pulpitis and dental loss.

Dentists should evaluate the patients' efficiency and autonomy in using oral care devices and suggest the use of electric toothbrush, chlorhexidine mouthwash twice a week. Regular check-ups and treatment with fluoride are also suggested (19). After having reached a good level of oral care, patients could initiate endodontic-conservative treatment with materials and techniques compatible with the disease.

\section{End of growth/Adult age}

Other surgical procedures are planned at the end of growth and concern the lower third of the skeleton and the ancillary procedures necessary to adjust the new skeletal architecture.

For surgical treatment of skeletal Class III accompanied by open bite, the use of standard techniques such as Le Fort I osteotomy and sagittal split osteotomy of the mandible (Fig. 8) is needed. It is important to note that the mandible is not particularly advanced and that both Class III and open bite are mainly due to maxilla malformation.

The Orthodontist in the pre and post surgical phase is essential in this age group.

Close monitoring of teeth eruption in Apert syndrome is necessary due to their need for individualized planning of dental element alignment (Fig. 9). Sometimes surgical interventions that provide individualizes osteotomies in order to normalize the architecture of the

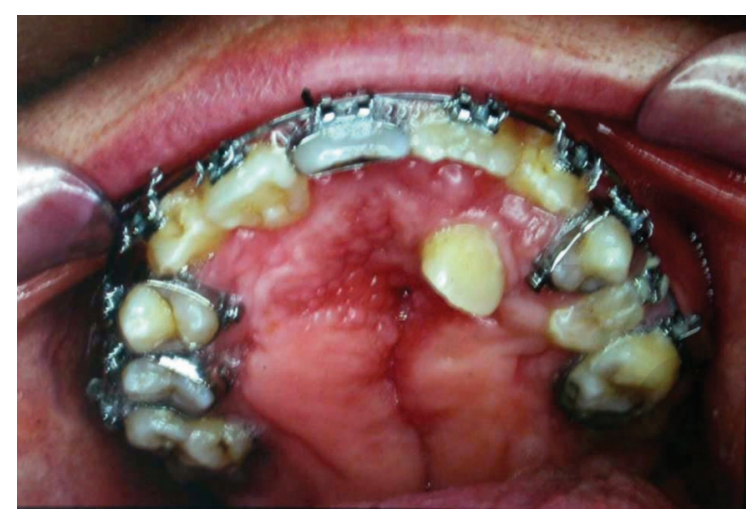

Figure 9. Orthodontic presurgical treatment. In the picture it could be noticed the presence of a palate incisor.

cranio-facial complex are combined with bone distraction method.

At the end of growth our surgical planning provides ancillary procedures necessary to adjust soft tissues and new skeletal architecture (rhinoplasty, lipofilling, plastic eyebrow, revision of the songs, autologous grafts, implants Medpor).

\section{Conclusions}

In conclusion it can be said that diagnostic and therapeutic planning in patients with Apert syndrome emphasizes the need to integrate various specialities. It is necessary to frame the patient's clinical picture upon his arrival.

Very important are also a multi-disciplinary plan and clinical programmed check-ups in accordance to a rational therapeutic clinical widely agreed upon and verified in its efficiency.

As noted in this paper establishing an integrated and tailored surgery timing, scheduling, combining and coordinating actions to be taken at different stages of the patient's age reduces the number of general anaesthesia thus simplifying therapy for both Apert patients and their families (Tab. 1).

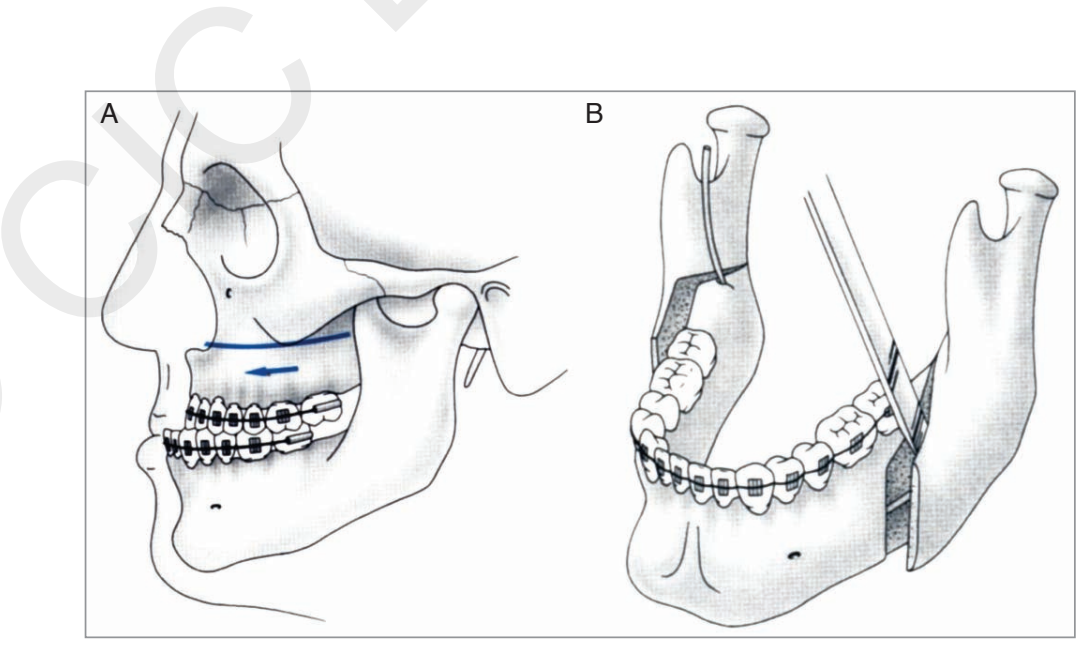

Figure 8. Inferior Third surgery: A: Le Fort I osteotomies and advancement; B: Bilateral split osteotomy of mandible. 
Table 1. Surgical timing in Apert syndrome.

\begin{tabular}{ll}
\hline Life period & Surgical treatment \\
\hline Within two yrs & Emergencies \\
& Cranioplasty \\
& Cleft Palate Correction \\
& Hand Surgery \\
\hline Growth period & Middle Third Correction \\
& Strabismus Correction \\
& Hand And Foot Surgery \\
& Orthodontic Treatment \\
\hline End of growth & Inferior Third Correction \\
& Individualized Surgery \\
& Ancillary Techniques \\
\hline
\end{tabular}

\section{References}

1. Ciurea AV, Toader C. Genetics of craniosynostosis: review of the literature. J Med Life. 2009 Jan-Mar;2(1):5-17.

2. Heuzé Y, Singh N, Basilico C, Jabs EW, Holmes G, Richtsmeier JT. Morphological comparison of the craniofacial phenotypes of mouse models expressing the Apert FGFR2 S252W mutation in neural crest- or mesoderm-derived tissues. Bone. 2014 Jun;63:101-9.

3. Goriely A, Wilkie. Paternal age effect mutations and selfish spermatogonial selection: causes and consequences for human disease. AO. Am J Hum Genet. 2012 Feb 10;90(2):175-200.

4. Cinalli G, Renier D, Sebag G, Sainte-Rose C, Arnaud E, Pierre-Kahn A. Chronic tonsillar herniation in Crouzon"s and Apert"s syndromes: the role of premature synostosis of the lambdoid suture. J Neurosurg. 1995;83:575-582.

5. Fearon JA, Podner C. Apert syndrome: evaluation of a treatment algorithm. Plastic and Reconstructive Surgery. 2013; 131:132-142.

6. Ileri Z, Goyenc YB. Apert syndrome: A case report. Eur J Dent. 2012;6(1):110-113.

7. Vadiati Saberi B, Shakoorpour A. Apert syndrome: report of a case with emphasis on oral manifestations. J Dent. 2011 Spring;8(2):90-95.

8. Moore MH. Upper airway obstruction in the syndromal craniosynostoses. Br J Plast Surg. 1993 Jul;46(5):355-362.

9. de Jong T, Toll MS, de Gier HH, Mathijssen IM. Audiological profile of children and young adults with syndromic and complex craniosynostosis. Arch. 2011 Aug;137(8):775778.

10. Allam KA, Wan DC, Khwanngern K, Kawamoto HK, Tanna $\mathrm{N}$, Perry A, et al. Treatment of apert syndrome: a long-term follow-up study. Plastic and Reconstructive Surgery. 2011;127:1601-1611.

11. Oberoi S, Hoffman WY, Vargervik K. Craniofacial team management in Apert. Am J Orthod Dentofacial Orthop. 2012; Vol. 141:S82-7.

12. Mazzone V. Apert's syndactyly: strategies in surgical treatment. Riv Chir Mano. 2006;2:124-127.

13. Mulliken JB, Bruneteau RJ. Surgical correction of the craniofacial anomalies in Apert syndrome. Clin Plast Surg.1991 Apr;18(2):277-289.

14. Posnick JC, Armstrong D, Bite U. Crouzon and Apert syndromes: intracranial volume measurements before and after cranio-orbital reshaping in childhood. Plast Reconstr Surg.1995 Sep;96(3):539-548.

15. Mulliken JB, Kaban LB, Evans CA, Strand RD, Murray JE. Facial skeletal changes following hypertelorbitism correction. Plast Reconstr Surg. 1986 Jan;77(1):7-16.

16. Dai J, Wang $X, Y u H$, Cheng J, Yuan H, Gui H, Shen S, Shen G. Simultaneous Le Fort I, II, and III osteotomies for correction of midface deficiency in Apert disease. J Craniofac Surg. 2012 Sep;23(5):1391-1395.

17. Nadal-López E, Gonzalez-Ramos J, Dogliotti PL, Routabul C, Zuccaro G. Simultaneous fronto-orbital advancement and dynamic posterior cranial vault expansion in Apert syndrome. J Craniofac Surg. 2012; 23(1):178-180.

18. Letra A, de Almeida AL, Kaizet R, Esper LA, Sgarbosa S, Granjeiro JM. Intraoral features of Apert's syndrome. Oral Surg Oral Med Oral Pathol Oral Radiol Endod. 2007 May;103(5):e38-4l.

19. Vadiati Saberi B, Shakoorpour A. Apert Syndrome: Report of a Case with Emphasis on Oral Manifestations. J Dent Tehran. 2011; Vol. 8(2):90-5. 